\title{
Cloud-Assisted Safe Driving Framework
}

\author{
Chia Hung Kao
}

\begin{abstract}
As the technologies for vehicular safety grow and mature, several advanced driving assisting systems are deployed to mitigate negative impacts on driving safety and protect drivers and passengers efficiently. However, the rate of fatalities and serious injuries in traffic accidents is still high. Based on the statistics from the Ministry of the Interior and National Highway Police Bureau in Taiwan, most critical accidents are caused by the circumstance that drivers do not aware the potential danger to their surroundings. Currently, several driving assisting systems deployed in vehicles can sense surrounding information for potential accidents prevention in time. In order to prevent potential accidents in advance for safer driving, a cloud-assisted safe driving framework is proposed in this paper. The framework leverages sensing devices in vehicles to acquire particular information of the front vehicles. The information will be acquired, recognized, and analyzed based on historical data preserved in the cloud. In addition, the information and comments on social network sites based on the recognized information will be collected and analyzed. Based on historical data and social information, a reputation for the recognized vehicle will be calculated in the cloud. Thus, corresponding suggestion based on the reputation is given to the drivers to perform defensive driving if necessary. Through the cloud-assisted safe driving framework, the driving safety can be achieved in advance efficiently.
\end{abstract}

Index Terms-Cloud computing, crowd sensing, reputation, driving safety.

\section{INTRODUCTION}

Recently, several advanced driving assisting systems, including driver fatigue monitoring system, lane departure warning system, collision warning system, blind-spot information system, and automatic emergency braking system, are developed and deployed to mitigate negative impacts on driving safety in order to protect drivers and passengers efficiently [1], [2]. Despite the fact that the technologies for vehicular safety grow and mature, based on several traffic reports worldwide, the rate of fatalities and serious injuries in traffic accidents is still high nowadays [3], [4].

In Taiwan, it was reported by the Ministry of the Interior that 239,168 accidents occurred from January to October in 2016, which caused 1,327 fatalities and 313,581 injured persons [5]. Furthermore, based on the statistics from the Ministry of the Interior, the circumstance that drivers do not aware the potential danger to their surroundings accounts for the highest proportion of causes of critical accidents. It was reported that within 1,286 critical traffic accidents, 290 accidents $(21.27 \%)$ are caused by the aforementioned

Manuscript received September 18, 2017; revised December 12, 2017. This work was partially supported by the Ministry of Science and Technology, R.O.C. under Grant 106-2218-E-143-001-.

Chia Hung Kao is with the Department of Applied Mathematics, National Taitung University, Taiwan (e-mail: chkao@nttu.edu.tw). circumstance. Similarly, based on the statistics from the National Highway Police Bureau in Taiwan, there were 67 critical traffic accidents in 2016, which caused 70 fatalities and 80 injured persons [6]. Within 67 critical accidents, 20 accidents $(29.85 \%)$ are caused by the same reason. Thus, in addition to the detection of driver status and vehicle behavior the detection of surrounding situations could be a key to provide proper information or alert to achieve safety driving efficiently.

Currently, collision warning system, blind spot information system, and automatic emergency braking system deployed in vehicles can sense surrounding information for potential accidents prevention in time. In order to prevent potential accidents in advance for safer driving, a cloud-assisted safe driving framework is proposed in this paper. The framework leverages smartphones or dash cams in vehicles to acquire particular information of the front vehicles [7]. The information of the front vehicles (e.g., license plate number) will be acquired and recognized in the cloud environment within the safe driving framework. Based on the recognized information, the historical data about the particular vehicle preserved in the cloud can be retrieved for further analysis. In addition, based on the recognized information, the information and comments about the particular vehicle contributed by crowd sensing on social networks, video sharing sites, or open data portals can be collected and analyzed. Based on historical data and social information, a reputation for the particular vehicle can be calculated by a specific analysis algorithm and reputation rules in the cloud. Thus, corresponding suggestion based on the reputation can be given to the drivers to perform defensive driving if necessary. Through the cloud-assisted safe driving framework, the driving safety can be achieved in advance efficiently.

This paper is organized as follows. Section II describes related work briefly. The design of the cloud-assisted safe driving framework is presented in Section III. Section IV introduces analysis algorithm and reputation rules used in the framework. Finally, Section V presents conclusion and future works.

\section{RELATED WORK}

\section{A. Driver Assisting Systems in Vehicles}

Vehicle manufacturers are developing and deploying various driver assisting systems in cars to protect drivers and passengers from potential danger [8]. Major driver assisting systems for safety can be classified into three categories.

The first one is to detect driver status, including fatigue, hypo-vigilance, or drunk, and provide alert to the corresponding drivers. Example assisting systems include driver fatigue monitoring system, driver hypo-vigilance 
system, and drunk-driving prevention system [9], [10].

The second one is to detect vehicle status, including speed, acceleration, brake, and lane position, to help drivers to maintain safe driving status. Example assisting systems include speed alert system, lane departure warning system, lane-keeping assisting system, and lane-centering assisting system [11].

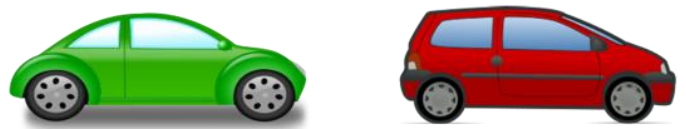

Vehicles with Sensing Devices

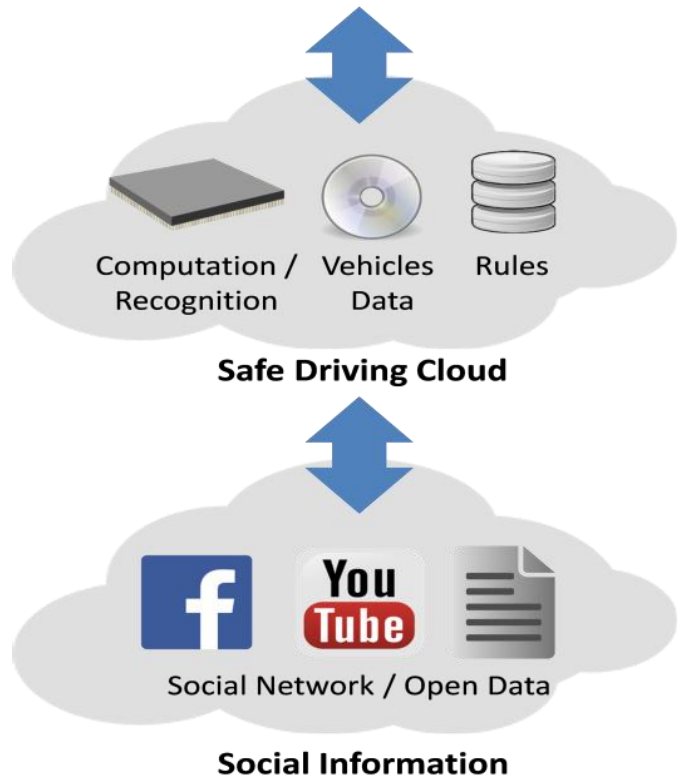

Fig. 1. Overview of the cloud-assisted safe driving framework.

The final one is to detect surrounding situations by lasers, radar, or cameras to provide alert information or automatically perform proper actions to prevent potential accidents in time. Example assisting systems include collision warning system, blind-spot information system, automatic emergency braking system, and adaptive cruise control system [12]. It is believed that more and more driver assisting systems will be developed and deployed in vehicles to improve driving safety continuously.

\section{B. Intelligent Transport Systems and Cloud Computing}

Intelligent Transport Systems (ITS) integrate advanced technologies, including computation, storage, communication, sensor, and data analytics, to build comprehensive infrastructure, platform, and innovative applications for transportation [13]. The goal of the ITS aims to increase the performance, effectiveness, efficiency, safety, and resilience of the system.

Besides, cloud computing has attracted considerable attention in recent years. It provides elastic computation and storage resources for rapid changing requirements. In addition, the service model brings benefits to integration among various services over different clouds.

Thus, several studies proposed innovative applications on intelligent transport systems based on the support of cloud computing technologies. For instance, Bitam and Mellouk proposed the ITS-Cloud for better road safety, transport productivity, travel reliability, informed travel choices, environment protection, and traffic resilience [14]. Peng, Li, $\mathrm{Li}$, and $\mathrm{Zhu}$ proposed a platform integrated with distributed storages and parallel computing technologies for traffic data management and analysis [15]. Based on the well-managed large amount of data by cloud computing technologies, further traffic or driving analysis can be achieved [16]. Finally, Dong et al. presented an intelligent framework based on IoT, $4 \mathrm{G}$, big data, cloud computing, and other novel ICT technologies [17].

\section{FRAMEWORK DESIGN}

In this section, the overview and the design of the cloud-assisted safe driving framework are introduced.

\section{A. Framework Overview}

Fig. 1 depicts the overview of the cloud-assisted safe driving framework. There are three major components in the framework, including vehicles with sensing devices, safe driving cloud, and social information. Each component is described in the following.

Vehicles with sensing devices: In order to improve the driving safety in advance based on the surrounding situation, the sensing devices such as smartphones and dash cams are deployed in vehicles. These devices help to acquire information of the front vehicles and transmit the acquired information to the safe driving cloud by the mobile network. Once the analysis results of the front vehicles are generated by the safe driving cloud, the smartphones, dash cams or other display devices can provide corresponding information or alert if defensive driving is necessary. In the near future, it is expected that the sensing devices and the network capability will be integrated and deployed into vehicles seamlessly.

Safe driving cloud: The safe driving cloud acquires, recognizes, and analyzes information transmitted from sensing devices deployed in vehicles. According to recognized information, the safe driving cloud retrieves related historical data and social information to compute a corresponding reputation by specific reputation rules. If the reputation is lower than a predefined threshold, the corresponding information or alert will be generated. For example, the license plate numbers of the front vehicles can be acquired by sensing devices and recognized by safe driving cloud. Then, associated historical data and social information (if available) can be used to calculate reputations for the front vehicles. If the reputations are not good enough, corresponding information or alert will be issued by the safe driving cloud to the drivers in order to prevent potential accidents in advance.

Social information: Social information includes information from different social networks, video sharing sites, and open data portals, which is contributed by crowd sensing [18]. For example, drivers may upload several videos recorded by smartphones and dash cams and give comments to the vehicles in the videos. Based on vehicles information and associated comments, the information can be used as the reference for driving advice. Currently, social networks, video sharing sites, and open data portals provide various 
APIs for efficient information retrieval and usage. Based on those APIs, the safe driving cloud can acquire social information for further driving behavior analysis to generate corresponding driving advice for drivers.

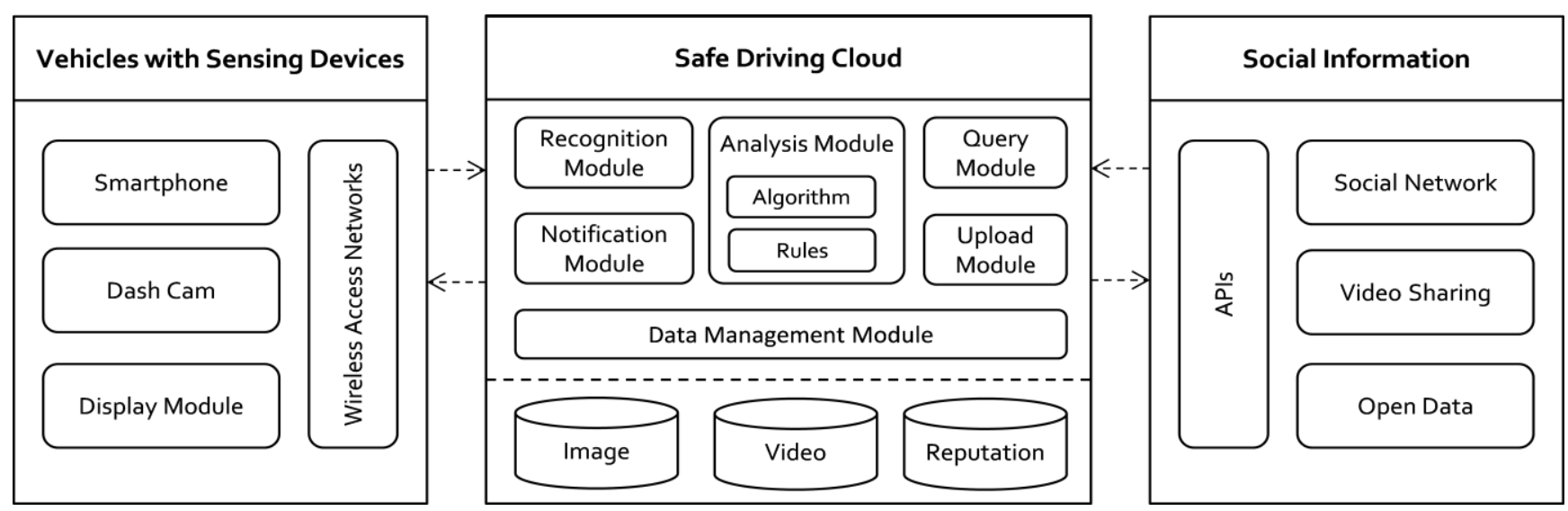

Fig. 2. Architecture design of the cloud-assisted safe driving framework.

\section{B. Framework Design}

Fig. 2 depicts the architecture design of the cloud-assisted safe driving framework. Modules in three major components, including vehicles with sensing devices, safe driving cloud, and social information, are introduced in the following.

1) Vehicles with sensing devices: Sensing devices can be smartphones, dash cams, and other devices integrated and deployed in vehicles, which can help to acquire images or videos of the front vehicles. The information will be transmitted to safe driving cloud continuously by the wireless access network, including $\mathrm{Wi}-\mathrm{Fi}, 4 \mathrm{G}$, and so on. In the near future, it is expected that the vehicle will be equipped with network connection capability. The sensing devices will also be integrated with vehicles seamlessly. Finally, a display module is used to present information of the front vehicles analyzed by safe driving cloud for drivers. Information, notifications or alerts will be provided through the display module based on the analysis results of the safe driving cloud. For example, alerts will be issued if the reputations of the front vehicles are lower than predefined threshold after analysis. Thus, drivers can perform corresponding actions based on the alerts to avoid potential accidents in advance.

2) Safe driving cloud: The safe driving cloud is responsible for the acquisition, preservation, management, and analysis of related driving information to achieve driving safety in advance. Major modules in the safe driving cloud include recognition module, notification module, analysis module, query module, upload module, data management module, image repository, video repository, and reputation repository.

Recognition Module: The recognition module is responsible for the recognition of the information acquired by the sensing devices deployed in vehicles. For example, the license plate numbers of the front vehicles can be recognized by the recognition module based on the images uploaded by sensing devices. The recognized license plate numbers can be used for further analysis. Based on the cloud computing infrastructure and technologies, computation capacity can be leveraged for large amounts of recognition tasks elastically and efficiently.

Notification Module: The notification module helps to generate and perform notifications to vehicles. Based on analysis results, corresponding information, notification, or alerts will be issued to display module deployed in vehicles.

Analysis Module: The analysis module is responsible for the analysis of available driving information to achieve driving safety in advance. There are two major components in the analysis module, including the analysis algorithm and reputation rules. Based on the recognized information of the front vehicles, the associated historical reputation data and available information gathered from social information will be retrieved for analysis module. Two sets of information are used by analysis module to calculate latest reputations of the front vehicles based on the analysis algorithm and reputation rules. The latest reputations will be further analyzed and used by notification module to perform notification tasks. Meanwhile, the refined reputation will be updated and preserved for analysis in the future.

Query Module: The query module helps to generate a specific query based on the information identified by recognition module to search social information preserved at social networks, video sharing sites, and open data portals. Through various APIs provided by social networks, video sharing sites, and open data portals, corresponding information retrieved from social information can be used for further analysis.

Upload Module: The upload module is responsible for uploading images or videos acquired by sensing devices deployed in vehicles. Through APIs provided by social networks, video sharing sites, and open data portals, the valuable images and videos can be shared to communities under the permission of drivers (also the users of the cloud-assisted safe driving framework).

Data Management Module: The data management module is responsible for the management and manipulation of image repository, video repository, and reputation repository. It also provides interface for other modules in the safe driving cloud to access necessary information for specific tasks.

Image Repository: The image repository helps to preserve and manage images acquired by the sensing devices deployed 
in vehicles. In addition, related metadata of the images, including timestamp and geographic information (if available) can be preserved. The images and associated metadata can be used for further analysis and community sharing. Storage infrastructure supported by cloud computing technologies is deployed to deal with the volume and velocity of generated images.

Video Repository: Similar to image repository, video repository helps to preserve and manage videos and associated metadata acquired by the sensing devices deployed in vehicles. The videos can be used for further analysis and community sharing. Cloud computing technologies are used to achieve the effective and efficient management of videos.

Reputation Repository: The reputation repository is responsible for the preservation and management of reputations about vehicles. The reputation is associated with recognition information and timestamp. Based on the recognized information, the corresponding reputation can be retrieved and used as the basis for further driving behavior analysis. After computation and analysis, the refined reputation will be updated to reputation repository for usage in the future.

3) Social information: Social information refers to various social networks, video sharing sites, and open data portals maintained and managed by different organizations or companies. The social information contributed by crowd sensing can be used by the cloud-assisted safe driving framework for further analysis. In addition to web or device interfaces for general users, APIs are exposed and provided by social networks, video sharing sites, and open data portals for further application integration and innovation. For example, YouTube APIs can let users search YouTube content, upload videos, play videos, get comments, and post comments to the specific video [19]. Similarly, Facebook Graph API can let users query data, post new articles, and upload images on the Facebook platform [20]. Last but not least, open data is getting momentum these years. Through the APIs of open data portals, valuable data can be acquired efficiently [21]. For instance, license plate number can be used to query the status of the particular vehicle from open data portal. Thus, in the cloud-assisted safe driving framework, if the information of the front vehicles can be acquired and recognized (e.g., license plate number), the information can be used as the query by the safe driving cloud to search related contents through APIs provided by social networks, video sharing sites, and open data portals. Related contents, including "title," "likes," "contents," "comments," "status," and so on, can be acquired through the query for further analysis. Besides, the images and videos recorded by sensing devices in vehicles can be uploaded by the safe driving cloud under the permission of drivers for community sharing.

\section{ANALYSIS ALgORITHM AND REPUTATION RULES}

In this section, the analysis algorithm and the reputation rules used by the cloud-assisted safe driving framework are introduced. The analysis algorithm is used to analyze and calculate reputations of the vehicles according to reputation rules based on the available driving information.

TABLE I: PARAMETERS USED IN THE ALGORITHM

\begin{tabular}{ll}
\hline \hline Parameter & Description \\
\hline Vehicle $V$ & $\begin{array}{l}\text { Vehicle } V \text { refers to the vehicle that acquires } \\
\text { information of the front vehicles with sensing devices }\end{array}$ \\
\hline Information $V i$ & $\begin{array}{l}\text { Information } V i \text { refers to the recognized information } \\
\text { (e.g., license plate number) acquired by sensing } \\
\text { devices deployed in Vehicle } V\end{array}$ \\
\hline Historical & $\begin{array}{l}\text { Historical reputation } R h \text { refers to the reputation of } \\
\text { particular vehicle preserved in reputation repository } \\
\text { based on recognized information } V i\end{array}$ \\
\hline $\begin{array}{l}\text { Social } R h \\
\text { information } S i\end{array}$ & $\begin{array}{l}\text { Social information } S i \text { refers to the information (e.g., } \\
\text { title, likes, comments, and status) retrieved from social } \\
\text { networks, video sharing sites, and open data portals by } \\
\text { searching recognized information } V i .\end{array}$ \\
\hline $\begin{array}{l}\text { Latest social } \\
\text { reputation } R s\end{array}$ & $\begin{array}{l}\text { Latest social reputation } R s \text { refers to reputation } \\
\text { calculated by social information } S i \text { based on } \\
\text { reputation rule }\end{array}$ \\
\hline $\begin{array}{l}\text { Refined } \\
\text { reputation } R r\end{array}$ & $\begin{array}{l}\text { Refined reputation } R r \text { refers to latest reputation } \\
\text { summarized by historical reputation } R h \text { and latest } \\
\text { social reputation } R s\end{array}$ \\
\hline \hline
\end{tabular}

Table I describes all the parameters used in the analysis algorithm and Algorithm 1 depicts the detailed information of the analysis algorithm.

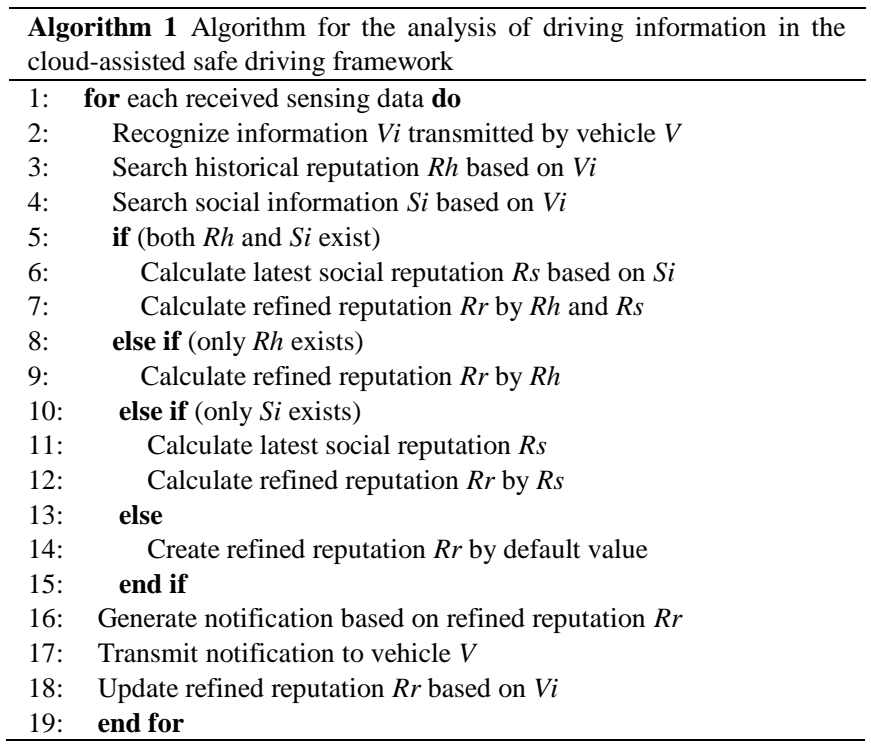

For each received sensing data from the vehicle, the recognition module helps to recognize the information (e.g., license plate number). Based on the available recognized information, the analysis module issues queries to reputation repository and the query module in the safe driving cloud. The social information preserved in social networks, video sharing sites, and open data portals will be searched through associated APIs. If historical reputation and associated social information with the recognized information are available, the analysis algorithm calculates social reputation based on available social information (e.g., content, likes, dislikes, and so on) according to reputations rules. The social reputation can be calculated based on the reputation rule (1). The weight of the factors can be tuned after simulation and actual deployment. New factors can also be added into the 
reputation rule to better present the reputation of driving behavior by social information. Besides, natural language processing techniques can be used to extract precise reputation from textual contents. Based on historical reputation and normalized social reputation, the refined reputation of the recognized vehicle can be calculated and updated.

$$
\begin{aligned}
R s= & a \times \text { likes }^{+} b \times \text { dislikes }^{+} \\
& c \times \text { comments }_{\text {positive }}+d \times \text { comments }_{\text {negative }}
\end{aligned}
$$

Secondly, if only historical reputation exists, the refined reputation will be calculated and updated based on the reputation rule (2). The reputation will change based on the configuration of parameter $k$. It can be used to simulate the reputation change after a certain period without any positive or negative driving behaviors.

$$
R r=(1+k) \times R h
$$

Thirdly, if only social information can be found, the analysis algorithm calculates social reputation based on available social information according to reputations rule (1). The result will be used as a refined reputation directly for the recognized vehicle.

Fourthly, if no historical reputation and no social information can be found, a default reputation (neutral) value for the recognized vehicle will be given and assigned based on the reputation rule. The default value can be adjusted after simulation and actual deployment.

Based on analysis algorithm and reputation rules, the refined reputation will be calculated. Thus, the information, notification or alert can be generated based on the value of the refined reputation. The corresponding information will be sent to the vehicle for the driver to perform proper actions immediately. Finally, the refined reputation of the recognized vehicle is updated to the reputation repository.

\section{CONCLUSION}

As the technologies for vehicular safety grow and mature, several advanced driving assisting systems are deployed to mitigate negative impacts on driving safety and protect drivers and passengers efficiently. Currently, collision warning system, blind spot information system, and automatic emergency braking system deployed in vehicles can sense surrounding information for potential accidents prevention in time. However, based on several traffic reports worldwide, the rate of fatalities and serious injuries in traffic accidents is still high nowadays.

In order to prevent potential accidents in advance for safer driving, a cloud-assisted safe driving framework is proposed in this paper. The framework leverages sensing devices in vehicles to acquire particular information of the front vehicles. The information of the front vehicles will be recognized and used to retrieve associated historical data and social information by safe driving cloud for further analysis. Based on historical data and available social information, a reputation for the recognized vehicle will be calculated by analysis algorithm and reputation rules proposed in this paper. Thus, corresponding suggestion based on the reputation is provided for the drivers to perform defensive driving if necessary. Through the cloud-assisted safe driving framework, the driving safety can be achieved in advance efficiently.

Future works include the implementation, simulation, and the evaluation of the cloud-assisted safe driving framework. The framework prototype will be implemented and deployed on smartphones and cloud environment built on OpenStack for the verification and validation of the framework functionalities. In addition, through the simulation, it helps to evaluate the analysis algorithm and the reputation rules for further tuning. Natural language processing (NLP) will also be used to precisely analyze information acquired from social network sites. Finally, the performance evaluation of the proposed framework will be performed to investigate performance characteristics and potential bottlenecks under the large scale of traffic situations.

\section{REFERENCES}

[1] R. Coppola and M. Morisio, "Connected car: Technologies, issues, future trends," ACM Computer Survey, vol. 49, no. 3, pp. 46:1-46:36, Oct. 2016.

[2] M. Pilipovic, D. Spasojevic, I. Velikic, and N. Teslic, "Toward intelligent driver-assist technologies and piloted driving: Overview, motivation and challenges," in Proc. the International Symposium on Industrial Electronics, Banja, Luka, 2014, pp. 10-14.

[3] U.S. Department of Transportation. (2010). National Highway Safety Administration. Traffic Safety Facts - Highlights of 2009 Motor Vehicle Crashes. [Online]. Available: http://www.nrd.nhtsa.dot.gov/pubs/811363.pdf

[4] World Health Organization. Road traffic deaths. [Online]. Available http://apps.who.int/gho/data/node.main.A997

[5] Ministry of the Interior. Traffic accident statistics. [Online]. Available: http://www.moi.gov.tw/stat/news_content.aspx?sn=11326

[6] National Highway Police Bureau. Traffic Accident Statistics. [Online] Available: http://www.hpb.gov.tw/files/11-1000-148.php.

[7] Z. Chen, J. Yu, Y. Zhu, Y. Chen, and M. Li, "D3: Abnormal driving behaviors detection and identification using smartphone sensors," in Proc. the 12th Annual IEEE International Conference on Sensing, Communication, and Networking, Seattle, WA, USA, 2015, pp. 524-532.

[8] K. H. Pathan and M. M. Patil, "Survey of cooperative advance driver assistance systems," in Proc. the 2016 International Conference on Control, Instrumentation, Communication and Computational Technologies, Kumaracoil, India, 2016, pp. 32-35.

[9] R. Chhabra, S. Verma, and C. R. Krishna, "A survey on driver behavior detection techniques for intelligent transportation systems," in Proc. the $20177^{\text {th }}$ International Conference on Cloud Computing, Dato Science \& Engineering - Confluence, Noida, India, 2017, pp. 36-41.

[10] Lee, S. Oh, S. Heo, and M. Hahn, "Drowsy driving detection based on the driver's head movement using infrared sensors," in Proc. the Second International Symposium on Universal Communication, Osaka, Japan, 2008, pp. 231-236.

[11] G. S. Tewolde, "Sensor and network technology for intelligent transportation systems," in Proc. the 2012 IEEE International Conference on Electro/Information Technology, Indianapolis, IN, USA, 2012, pp. 1-7.

[12] W. Song, M. Fu, Y. Yang, M. Wang, X. Wang, and A. Kornhauser, "Real-time lane detection and forward collision warning system based on stereo vision," in Proc. the 2017 IEEE Intelligent Vehicles Symposium, Los Angeles, CA, USA, 2017, pp. 493-498.

[13] M. Alam, J. Ferreira, and J. Fonseca, "Introduction to intelligent transportation systems," Intelligent Transportation Systems. Studies in Systems, Decision and Control, vol. 52, Springer, Cham, 2016, pp. $1-17$.

[14] S. Bitam and A. Mellouk, "ITS-cloud: Cloud computing for Intelligent transportation system," in Proc. the 2012 IEEE Global Communications Conference, Anaheim, CA, USA, 2012, pp. 2054-2059. 
[15] W. Peng, Y. Li, B. Li, and X. Zhu, "An analysis platform of road traffic management system log data based on distributed storage and parallel computing techniques," in Proc. the 2016 IEEE International Conference on Big Data and Cloud Computing, Social Computing and Networking, Sustainable Computing and Communications, Atlanta, GA, USA, 2016, pp. 585-590.

[16] M. Zhang and C. Chen, T. Wo, T. Xie, M. Z. A. Bhuiyan, and X. Lin "SafeDrive: Online driving anomaly detection from large-scale vehicle data," IEEE Transactions on Industrial Informatics, vol. 13, no. 4, pp. 2087-2096, Aug. 2017

[17] X. Dong, J. Zhou, B. Hu, J. Riekki, G. Xiong, F. Wang, and F. Zhu, "A framework of future Innovative urban transport," in 2016 IEEE 19th International Conference on Intelligent Transportation Systems, Rio de Janeiro, Brazil, 2016, pp. 19-23.

[18] Q. Liu, S. Kumar, and V. Mago, "SafeRNet: Safe Transportation Routing in the era of Internet of Vehicles and Mobile Crowd Sensing," in Proc. the 2017 4th IEEE Annual Consumer Communications \& Networking Conference, Las Vegas, NV, USA, 2017, pp. 299-304.

[19] Google Developers. YouTube Data API. [Online]. Available: https://developers.google.com/youtube/v3/
[20] Facebook. Facebook for Developers. [Online]. Available: https://developers.facebook.com/

[21] A. Zuiderwijk, M. Janssen, and A. Parnia, "The complementarity of open data infrastructures: an analysis of functionalities," in Proc. the 14th Annual International Conference on Digital Government Research, Quebec, Canada, 2013, pp.166-171.

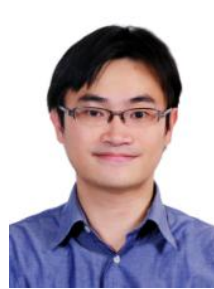

Chia Hung Kao received his Ph.D. degree in the Department of Electrical Engineering, National Cheng Kung University, Taiwan, in 2009. After getting his degree, he joined Institute for Information Industry (III) as a senior engineer and participated in the development of several cloud computing and big data analytics products. Since 2015, he joined National Taitung University as an assistant professor in the department of applied mathematics. His research interests include software engineering, cloud computing, and big data analytics. 